Arch. Tierz., Dummerstorf 49 (2006) 3, 250-258

Lehr- und Versuchsgut Oberschleißheim der Tierärztlichen Fakultät, Ludwig-Maximilians-Universität, München

PRISCA KREMER, STEFAN NÜSKE, ARMIN M. SCHOLZ und MARTIN FÖRSTER

\title{
Einfluss von elastischem Bodenbelag auf Milchmenge, Fett, Eiweiß und Zellgehalt bei Kühen in Laufstallhaltung
}

\author{
Herrn Professor Dr. Gerhard Seeland zum 65. Geburtstag gewidmet
}

\begin{abstract}
Title of the paper: Effect of soft flooring in free stalls on milk yield, fat, protein and somatic cell counts in dairy cattle

Research conducted at the Experimental Farm Oberschleißheim focused on comparing milk yield, fat, protein and somatic cell counts in dairy cattle housed in free stalls with concrete slatted floor and rubber mats covering the concrete slatted floor. Data were collected from 49 cows in 53 lactation periods, which were divided into experimental group $(n=26)$ on rubber matted floor $(E B G)$ and control group $(n=27)$ on concrete slatted floor (BBG). Housing conditions of both groups differed in kind of cubicles. EBG had soft rubber mat mattresses, BBG had cubicles with straw manure mattresses. Data analysed originated from the official milk performance testing of the "Landeskuratorium der Erzeugerringe für tierische Veredelung in Bayern e.V." and from activity counters worn by the cows. Statistical analysis resulted in a significant higher activity level of the EBG versus BBG. Milk yield and protein showed no significant difference between both groups, fat was significant higher in BBG. Somatic cell counts were significant lower in EBG. Data analysed regarding activity level classes showed a trend of decreasing milk yield and milk constituents with increasing activity. No evidence was found that soft floor results in higher milk yield. But a higher activity level of EBG cows might indicate a higher cow comfort on elastic floor.
\end{abstract}

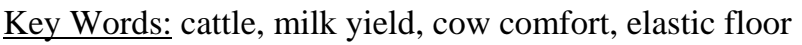

\section{Zusammenfassung}

Ziel der vorliegenden Studie war es, den Einfluss eines elastischen Bodens im Laufstall auf Milchleistung, Fettgehalt, Fettmenge, Eiweißgehalt, Eiweißmenge und Zellzahlen zu untersuchen. Hierzu wurden 49 Kühe in 53 Laktationen (jew. 305 d) in zwei Gruppen aufgeteilt. Die Versuchsgruppe (EBG) $(n=26)$ wurde auf Betonspaltenboden mit elastischen Auflagen, die Kontrollgruppe (BBG) $(n=27)$ auf Betonspaltenboden ohne Auflagen aufgestallt. Beide Abteile unterschieden sich durch die Art der Liegeboxen. Der EBG standen Hochboxen, der BBG Tiefboxen zur Verfügung. Zur Auswertung kamen Daten der Milchleistungsprüfung des Landeskuratorium der Erzeugerringe für tierische Veredelung in Bayern e.V. und Daten von Bewegungszählern, die die Tiere am Halsband trugen. Die Auswertung ergab ein signifikant höheres Aktivitätslevel der EBG gegenüber der BBG. Bei der Auswertung der Milchdaten lagen keine signifikanten Unterschiede bei Milchmenge, Eiweißgehalt und Eiweißmenge vor. Fettgehalt und Fettmenge lagen in der BBG signifikant höher als in der EBG. Der Zellgehalt lag in der EBG signifikant niedriger als in der BBG. Eine Auswertung getrennt nach Aktivitätslevel zeigte einen Trend abnehmender Leistung mit steigender Aktivität. Demnach konnte im Rahmen dieser Studie keine Leistungsverbesserung der Tiere durch den Einfluss des elastischen Bodens bewiesen werden. Das signifikant höhere Aktivitätslevel der EBG spricht aber für ein erhöhtes Maß an Kuhkomfort durch den weichen Boden.

Schlüsselwörter: Rind, Milchleistung, Kuhkomfort, elastische Bodenbeläge

\section{Einleitung}

Die moderne Milchviehhaltung wird durch einen enormen wirtschaftlichen Druck belastet. Der sinkende Milchpreis stellt für zahlreiche Betriebe eine existentielle Bedro- 
hung dar. Daher stehen neben gezielter Ausschöpfung des genetischen Potentials der Tiere, optimierten Futterrationen und modernster Melktechnik längst auch Bemühungen um Schadensvermeidung und maximale Bedarfsdeckung der Tiere im Vordergrund. Im Rahmen dieser Entwicklung hat der Begriff des Kuhkomforts zunehmend an Bedeutung gewonnen. Es liegen zahlreiche Untersuchungsergebnisse zu diesem Themenkomplex vor. Meist handelt es sich hierbei um Untersuchungen zur Optimierung von Liegeflächen bzgl. Abmessungen, Komfort und Hygiene, Verhaltensstudien, Verschmutzungsgrad der Euter und bestimmter Körperareale, Studien zum Stallklima oder der Bodenbeschaffenheit in Ställen.

Letztere wecken zunehmend das Interesse beteiligter Fachkreise, da hier die Etablierung von elastischen Bodenbelägen in Laufställen stattgefunden hat. Diese zeichnen sich dadurch aus, dass bei darauf gehaltenen Kühen Komfort-, Brunst-, Sozial- und Lokomotionsverhalten als bedarfsdeckend bewertet werden können (WOLF, 2000; BENZ, 2002; WANDEL et al., 2002; PARTES, 2004).

Umstritten ist derzeit die Schadensvermeidung durch elastische Bodenbeläge. Während BERGSTEN (2004) von mehr als 20 Jahren positiven Erfahrungen mit elastischen Bodenbelägen berichtet und sich dabei auf Ergebnisse von Studien bezieht, die eine verbesserte Klauengesundheit von Tieren, gehalten auf elastischen Bodenbelägen, belegen (BERGSTEN, 1994; BERGSTEN und FRANK, 1996; HULTGREN und BERGSTEN, 2001; BENZ, 2002), häufen sich in letzter Zeit Ergebnisse, die diesen Effekt in Frage stellen. BAHRS (2005) berichtet von einem gehäuften Auftreten von Dermatitis Digitalis bei Mastbullen, die auf elastischen Bodenbelägen gehalten werden. SAMEL et al. (2005) können beim Vergleich von Kühen auf Betonspaltenboden mit Kühen auf elastischen Bodenbelägen keinen Unterschied bei der Inzidenz bestimmter Klauenerkrankungen feststellen, berichten aber in Übereinstimmung mit KREMER et al. (2004) von einem verminderten Abrieb des Hornes auf elastischem Boden und einer daraus resultierenden Deformation des Hornschuhs. Da der Effekt des elastischen Bodens auf die Klauengesundheit somit als umstritten angesehen werden kann, liegt der Gedanke nah, den Grad des Kuhkomforts eines solchen Bodens über die Milchleistung der Kühe zu beurteilen. In der Literatur finden sich nahezu keine Untersuchungen, die einen direkten Schluss von verbessertem Kuhkomfort auf verbesserte Milchleistung zulassen. BENZ (2002) berichtet im Rahmen ihrer Untersuchungen zum Verhalten und zur Klauengesundheit von Kühen auf Betonspaltenboden und auf Spaltenböden mit elastischen Auflagen von einem Anstieg der Milchleistung der Tiere auf elastischem Boden. Statistisches Material liegt hierzu jedoch nicht vor, da es nicht Gegenstand der Untersuchung war, sondern lediglich ein Begleiteffekt.

Ziel der nachfolgenden Untersuchung war es daher herauszufinden, ob allein durch das Verlegen eines elastischen Bodens in einem Laufstall die Milchleistung der Herde gesteigert werden kann.

\section{Material und Methode}

Tiere

Die Untersuchungen wurden an 49 F1-Kreuzungskühen der Rassen Deutsche Holsteins (DH) und Deutsches Fleckvieh (FV) durchgeführt. Hierbei lag 19 mal der Genotyp FV-DH vor und 30 mal der Genotyp DH-FV, wobei jeweils die erstgenannte Rasse die Vaterrasse bezeichnet. Zur Auswertung kamen Daten aus 30 Erst- und 23 
Zweitlaktationen dieser 49 Tiere. Die Verteilung der Tiere auf Versuchs- und Kontrollgruppe einschließlich der Genotypen und Laktationen ist in Tabelle 1 dargestellt.

Tabelle 1

Verteilung der Tiere nach Genotyp und Laktation auf Versuchs- und Kontrollgruppe (Distribution of cows within the study into experimental and controll group, divided in genotypes and lactation)

\begin{tabular}{l|llllll}
\hline \multicolumn{1}{l}{ Laktation } & $\begin{array}{l}\text { EBG } \\
\text { DH-FV }\end{array}$ & FV-DH & Gesamt & $\begin{array}{l}\text { BBG } \\
\text { DH-FV }\end{array}$ & FV-DH & Gesamt \\
\hline 1 & 9 & 5 & 14 & 10 & 6 & 16 \\
2 & 7 & 5 & 12 & 7 & 4 & 11 \\
\hline Summe & 16 & 10 & 26 & 17 & 10 & 27 \\
\hline
\end{tabular}

EBG = Elastischer Boden Gruppe / rubber matted floor group, BBG = Betonboden Gruppe / concrete slatted floor group, DH = Deutsche Holsteins / German Holstein, FV = Deutsches Fleckvieh / German Fleckvieh

\section{Versuchsaufbau und -durchführung}

Die Untersuchung fand im Zeitraum von November 2002 bis März 2005 am Lehr- und Versuchsgut der Universität München statt. Der dortige Laufstall ist in zwei Abteile aufgeteilt, die mit Betonspaltenboden ausgestattet sind. In einem Abteil stehen den Tieren Tiefboxen mit Häckselstroheinstreu zur Verfügung, das andere Abteil verfügt über Hochboxen mit Gummimatten. In jedem Abteil stehen 63 Liegeplätze zur Verfügung. Das Hochboxenabteil ist mit Futterwannen mit integrierter Grundfutterverwiegung ausgestattet, die eine Fressplatzanzahl von 28 bedingen. Das Tiefboxenabteil verfügt über ein Selbstfangfressgitter mit 54 Fressplätzen. Die Belegungsdichte war während der gesamten Versuchszeit in beiden Abteilen annähernd gleich mit ca. 48 Tieren. Das Hochboxenabteil wurde vor Versuchsbeginn mit exakt auf die Spaltenmaße zugeschnittenen Gummimatten der Fa. Gummiwerk Kraiburg ausgestattet. Drei Monate zuvor wurden die Liegeflächen in diesem Abteil mit besonders komfortablen Gummimatten optimiert, um dem Problem des Spaltenliegens vorzubeugen und den gleichen Liegekomfort wie in den Tiefstreuboxen im Betonbodenabteil zu erreichen. Beide Abteile verfügen über ein Automatisches Melksystem (AMS) vom Typ „Astronaut“ der Fa. Lely. Zweimal täglich wurde den Tieren eine TMR, bestehend aus Grassilage, Maissilage, Heu, Körnermais, Rapsschrot, Sojaschrot und Kraftfutter vorgelegt. Der Rationsberechnung lagen 700 kg Lebendgewicht, 27 kg Tagesmilchleistung bei 4,0\% Fett und 3,4\% Eiweiß zugrunde. Der Energiegehalt der TMR betrug durchschnittlich 6,85 MJ NEL/kg TM, der RP-Gehalt lag bei 18,4 \%. Zusätzlich erfolgte eine leistungsbezogene Kraftfutterzuteilung im AMS (Tab. 2). Der Energiegehalt des Kraftfutters betrug 8,5 MJ NEL /kg TM.

Tabelle 2

Leistungsbezogene Kraftfutterzuteilung im automatischen Melksystem (Performance-orientated portioning of concentrate in the automatic milking system)

\begin{tabular}{l|llllllllll}
\hline Tägliche & bis & bis & bis & bis & bis & bis & bis & bis & bis & bis \\
Milchleistung $(\mathrm{kg})$ & 15 & 20 & 25 & 30 & 35 & 40 & 45 & 50 & 55 & 60 \\
\hline $\begin{array}{l}\text { Tägliche Kraft- } \\
\text { futtermenge }(\mathrm{kg})\end{array}$ & 1 & 2 & 3 & 4 & 5 & 6 & 7 & 8 & 9 & 10 \\
\hline
\end{tabular}

Die Tiere wurden 21 Tage vor dem erwarteten Abkalbetermin, einer zufälligen Verteilung auf Versuchsgruppe auf elastischem Boden (EBG) und Kontrollgruppe auf 
Betonboden (BBG) folgend, eingestallt. Die Tiere wurden mit Responderhalsbändern ausgestattet, die der Erkennung im AMS dienen und gleichzeitig einen Aktivitätszähler (AM-activity-collar, Fa. Nedap-agri) beinhalten, der ebenfalls am AMS abgelesen wurde. Das Zählwerk des Aktivitätsmessgerätes zählt die Halsbewegungen der Tiere und steigt mit jeder 40. Bewegung um die Einheit „1“.

Um einen einheitlich guten Klauenstatus zu gewährleisten und Leistungseinbußen durch schmerzhafte Klauenerkrankungen zu minimieren wurden alle Tiere des Versuchs am Tag des Einstallens, am Tag 150 und am Tag 305 der Laktation einer Funktionellen Klauenpflege unterzogen. Die Daten der Bewegungsaktivität wurden zuerst separat ausgewertet und anschließend in die Auswertung der Milchdatenanalyse mit einbezogen. Die analysierten Milchdaten stammen aus der unabhängigen, 11 x jährlich durchgeführten Milchkontrolle des Landeskuratorium der Erzeugerringe für tierische Veredelung in Bayern e.V. (LKV). In die Analyse wurde die Milchmenge in kg, der Fettgehalt in \%, der Eiweißgehalt in \% und die sich daraus ergebenden Fett- und Eiweißmengen in kg und die Zellzahl mit einbezogen. Es wurden alle Milchprüfungen bis zum 305. Laktationstag ausgewertet.

\section{Statistische Bearbeitung}

Für die statistische Aufarbeitung des Datenmaterials wurde mittels des Softwarepakets SAS $^{\circledR}$ (Version 8.2) unter Verwendung eines generalisierten linearen Modells (GLM) eine Varianzanalyse durchgeführt. Das folgende Modell wurde für die Berechnung der Bewegungsaktivität verwendet:

$\begin{array}{ll}\mathrm{y}_{\mathrm{ijklmno}}=\mu+\mathrm{G}_{\mathrm{i}}+\mathrm{A}_{\mathrm{j}}+\mathrm{G}_{\mathrm{i}} * \mathrm{~A}_{\mathrm{j}}+\mathrm{F}_{\mathrm{k}}+\mathrm{L}_{\mathrm{l}}+\mathrm{J}_{\mathrm{m}} * \mathrm{Q}_{\mathrm{n}}+\mathrm{e}_{\mathrm{ijklmno}} \\ \mathrm{y}_{\mathrm{ijklmno}}= & \text { Beobachtungswert } \\ \mu= & \text { Erwartungswert von y } \\ \mathrm{G}_{\mathrm{i}}= & \text { Genotyp }(\mathrm{i}=1,2) \\ \mathrm{A}_{\mathrm{j}}= & \text { Aufstallung }(\mathrm{j}=1,2) \\ \mathrm{G}_{\mathrm{i}} * \mathrm{~A}_{\mathrm{j}}= & \text { Interaktion } \mathrm{G} * \mathrm{~A}(\mathrm{i} * \mathrm{j}) \\ \mathrm{F}_{\mathrm{k}}= & \text { Kraftfutterklassen }(\mathrm{k}=1,2,3,4,5,6,7) \\ \mathrm{L}_{\mathrm{l}}= & \text { Laktationsnummer }(\mathrm{l}=1,2) \\ \mathrm{J}_{\mathrm{m}} * \mathrm{Q}_{\mathrm{n}}= & \text { Interaktion Jahr }(\mathrm{m}=1,2) * \text { Quartal }(\mathrm{n}=1,2,3,4) \\ \mathrm{e}_{\mathrm{ijklmno}}= & \text { Restfehler }\end{array}$

Für die Auswertung der Bewegungsaktivität wurden die täglichen Aktivitätswerte der Tiere logarithmiert (ln), um eine Normalverteilung des Merkmals zu erhalten. Die Analyse der Milchdaten wurde mittels folgendem Modell vorgenommen:

$$
\begin{aligned}
& \mathrm{y}_{\mathrm{ijklmnop}}=\mu+\mathrm{G}_{\mathrm{i}}+\mathrm{A}_{\mathrm{j}}+\mathrm{G}_{\mathrm{i}} * \mathrm{~A}_{\mathrm{j}}+\mathrm{F}_{\mathrm{k}}+\mathrm{B}_{\mathrm{l}}+\mathrm{L}_{\mathrm{m}}+\mathrm{J}_{\mathrm{n}} * \mathrm{Q}_{\mathrm{o}}+\mathrm{e}_{\mathrm{ijklmnop}} \\
& \mathrm{y}_{\mathrm{ijklmnop}}=\quad \text { Beobachtungswert } \\
& \mu=\quad \text { Erwartungswert von } \mathrm{y} \\
& \mathrm{G}_{\mathrm{i}}=\quad \text { Genotyp }(\mathrm{i}=1,2) \\
& A_{j}=\quad \text { Aufstallung }(j=1,2) \\
& \mathrm{G}_{\mathrm{i}} * \mathrm{~A}_{\mathrm{j}}=\text { Interaktion } \mathrm{G} * A(\mathrm{i} * \mathrm{j}) \\
& \mathrm{F}_{\mathrm{k}}=\quad \text { Kraftfutterklassen }(\mathrm{k}=1,2,3,4,5,6,7) \\
& \mathrm{B}_{\mathrm{l}}=\quad \text { Bewegungsaktivitätsklasse }(\mathrm{l}=1,2,3,4,5)
\end{aligned}
$$


$\mathrm{L}_{\mathrm{m}}=\quad$ Laktationsnummer $(\mathrm{m}=1,2)$

$\mathrm{J}_{\mathrm{n}} * \mathrm{Q}_{\mathrm{o}}=\quad$ Interaktion Jahr $(\mathrm{n}=1,2) *$ Quartal $(\mathrm{o}=1,2,3,4)$

$\mathrm{e}_{\mathrm{ijklmnop}}=$ Restfehler

Da im Jahr 2002 für das letzte Quartal nur ein Datensatz eines Tieres vorlag bzw. im Jahr 2005 im ersten Quartal nur insgesamt drei Datensätze vorlagen, wurden diese Datensätze jeweils dem darauf folgenden, bzw. vorhergehenden Quartal zugeordnet. Daraus ergeben sich zwei Prüfjahre mit je vier Quartalen. Bei der Aufteilung in Kraftfutterklassen wurden ebenso die Klassen (Tab. 2), die nur Einzeltiere enthielten der nächst höheren, bzw. nächst niedrigeren Klasse zugeordnet. Somit wurden Tiere, die täglich $<3 \mathrm{~kg}$ Kraftfutter erhielten in der ersten Gruppe und Tiere mit $\geq 8 \mathrm{~kg}$ Kraftfutter in der siebenten Gruppe zusammengefasst. Die Bewegungsaktivitätsklassen wurden gebildet, indem die Tiere ihrer durchschnittlichen täglichen Aktivität zwischen zwei Milchprüfterminen nach in fünf Klassen eingeteilt wurden. Wobei Klasse 1 Tiere beinhaltete, die eine tägliche Bewegungsaktivität von $<10$ aufwiesen, Klasse 210 - 19, Klasse 320 - 29, Klasse 430 - 39 und Klasse $5 \geq 40$. Die Zellzahlen wurden für die Auswertung, wie die Bewegungsaktivitätszahlen logarithmiert (ln). Die Grenze für die Signifikanz der kleinsten Quadrate Mittelwerte wurde bei p $<0,05$ festgelegt.

\section{Ergebnisse}

Den Ergebnissen vorangestellt sei die Auswertung der Bewegungsaktivität der Tiere. Hier zeigt sich ein signifikanter Unterschied zwischen den beiden Aufstallungssystemen (Tab. 3). Die Tiere der EBG zeigen ein signifikant höheres Aktivitätslevel als die Tiere der BBG.

\section{Tabelle 3}

Durchschnittliche tägliche Bewegungsaktivität (ln) der Tiere in Versuchs- und Kontrollgruppe (kleinste Quadrate Mittelwerte \pm Standardschätzfehler) (Mean daily activity of the cows depending on flooring systems (Least Squares Means \pm Standard Error of Estimation))

\begin{tabular}{l|lll}
\hline Aufstallungsform & EBG & $B B G$ & Prüfkriterium \\
\hline \multirow{3}{*}{ Bewegungsaktivität (ln) } & 3,11 & 2,87 & $\mathbf{p}<\mathbf{0 , 0 0 0 1}$ \\
& $\pm 0,01$ & $\pm 0,01$ & \\
\hline
\end{tabular}

EBG = Elastischer Boden Gruppe / rubber matted floor group, BBG = Betonboden Gruppe / concrete slatted floor group

Die Auswertung der Milchprüfdaten der Versuchs- und Kontrollgruppe erbrachte keinen signifikanten Unterschied in der durchschnittlichen täglichen Milchmenge, dem Eiweißgehalt und der sich daraus ergebenden Eiweißmenge (Tab. 4).

Bei Fettgehalt und Fettmenge wurde ein signifikanter Unterschied zwischen beiden Gruppen ersichtlich. Die Leistung der BBG lag bzgl. des Fettgehaltes 0,29 \% über der Leistung der EBG ( $<<0,0001)$. Bei der Fettmenge entspricht dies einem Unterschied von 90g täglich ( $p=0,0002)$. Die Zellzahlen lagen in der EBG signifikant niedriger als in der BBG $(p<0,0001)$. Bei der Auswertung der Daten getrennt nach Aktivitätsklassen (Tab. 5) wurde ersichtlich, dass die Tiere tendenziell mit steigender Aktivität geringere Leistungen zeigen. In der Aktivitätsklasse 1 zeigen die Tiere die höchste Milchmenge, den höchsten Fettgehalt, die höchste Eiweißmenge und den niedrigsten Zellgehalt. Die Unterschiede sind zwar nicht statistisch signifikant, jedoch ist dieser Trend deutlich zu beobachten. Eine Ausnahme bildet das Merkmal 
Eiweißgehalt. Es stellt mit 3,63 \% in der Aktivitätsklasse 3 den Spitzenwert dar und liegt damit 0,06 \% über dem Eiweißgehalt der Aktivitätsklasse 1 und 2.

Tabelle 4

Durchschnittliche tägliche Milchmenge, -inhaltsstoffe und Zellgehalt der Kühe auf Betonboden und auf elastischem Boden (kleinste Quadrate Mittelwerte \pm Standardschätzfehler) (Mean daily milk yield, milk constituents and somatic cell counts depending on flooring systems (Least Squares Means \pm Standard Error of Estimation))

\begin{tabular}{l|lll}
\hline & $E B G$ & $B B G$ & Prüfkriterium \\
\hline Milchmenge (kg) & 28,52 & 28,89 & $\mathrm{p}=0,5517$ \\
& $\pm 0,54$ & $\pm 0,54$ & \\
Fettgehalt (\%) & 3,40 & 3,69 & $\mathbf{p}<\mathbf{0 , 0 0 0 1}$ \\
& $\pm 0,05$ & $\pm 0,05$ & \\
Fettmenge (kg) & 0,95 & 1,04 & $\mathbf{p}=\mathbf{0 , 0 0 0 2}$ \\
& $\pm 0,02$ & $\pm 0,02$ & \\
Eiweißgehalt (\%) & 3,56 & 3,54 & $\mathrm{p}=0,4558$ \\
& $\pm 0,03$ & $\pm 0,03$ & \\
Eiweißgehalt (kg) & 1,00 & 1,01 & $\mathrm{p}=0,7942$ \\
& $\pm 0,02$ & $\pm 0,02$ & $\mathbf{p}<\mathbf{0 , 0 0 0 1}$ \\
\hline
\end{tabular}

EBG = Elastischer Boden Gruppe / rubber matted floor group, BBG = Betonboden Gruppe / concrete slatted floor group

Tabelle 5

Durchschnittliche tägliche Milchmenge, -inhaltsstoffe und Zellzahlen der Kühe, aufgeteilt nach Aktivitätsklassen (kleinste Quadrate Mittelwerte \pm Standardschätzfehler) (Mean daily milk yield, milk constituents and somatic cell counts divided into activity classes (Least Squares Means \pm Standard Error of Estimation))

\begin{tabular}{|c|c|c|c|c|c|}
\hline Aktivitätsklasse & 1 & 2 & 3 & 4 & 5 \\
\hline Milchmenge (kg) & $\begin{array}{l}30,16^{\mathrm{a}} \\
\pm 0,87\end{array}$ & $\begin{array}{l}29,12^{\text {ab }} \\
\pm 0,51\end{array}$ & $\begin{array}{l}27,71^{b} \\
\pm 0,76\end{array}$ & $\begin{array}{l}27,71^{\text {ab }} \\
\pm 1,22\end{array}$ & $\begin{array}{l}28,83^{\text {ab }} \\
\pm 0,81\end{array}$ \\
\hline Fettgehalt (\%) & $\begin{array}{l}3,66 \\
\pm 0,09\end{array}$ & $\begin{array}{l}3,64 \\
\pm 0,05\end{array}$ & $\begin{array}{l}3,50 \\
\pm 0,08\end{array}$ & $\begin{array}{l}3,48 \\
\pm 0,12\end{array}$ & $\begin{array}{l}3,46 \\
\pm 0,08\end{array}$ \\
\hline Fettmenge (kg) & $\begin{array}{l}1,09^{\mathrm{a}} \\
\pm 0,03\end{array}$ & $\begin{array}{l}1,04^{\mathrm{ac}} \\
\pm 0,02\end{array}$ & $\begin{array}{l}0,94^{\mathrm{b}} \\
\pm 0,03\end{array}$ & $\begin{array}{l}0,95^{\text {bc }} \\
\pm 0,05\end{array}$ & $\begin{array}{l}0,97^{\mathrm{bc}} \\
\pm 0,03\end{array}$ \\
\hline Eiweißgehalt (\%) & $\begin{array}{l}3,57^{\mathrm{ab}} \\
\pm 0,04\end{array}$ & $\begin{array}{l}3,57^{\mathrm{ab}} \\
\pm 0,02\end{array}$ & $\begin{array}{l}3,63^{\mathrm{a}} \\
\pm 0,04\end{array}$ & $\begin{array}{l}3,46^{\mathrm{b}} \\
\pm 0,06\end{array}$ & $\begin{array}{l}3,51^{\mathrm{b}} \\
\pm 0,04\end{array}$ \\
\hline Eiweißmenge (kg) & $\begin{array}{l}1,07^{\mathrm{a}} \\
\pm 0,03\end{array}$ & $\begin{array}{l}1,03^{\mathrm{ac}} \\
\pm 0,02\end{array}$ & $\begin{array}{l}0,98^{\mathrm{bc}} \\
\pm 0,02\end{array}$ & $\begin{array}{l}0,95^{\mathrm{b}} \\
\pm 0,04\end{array}$ & $\begin{array}{l}1,00^{\mathrm{bc}} \\
\pm 0,03\end{array}$ \\
\hline Zellgehalt (ln) & $\begin{array}{l}3,85^{\mathrm{a}} \\
\pm 0,13\end{array}$ & $\begin{array}{l}4,28^{\mathrm{bc}} \\
\pm 0,08\end{array}$ & $\begin{array}{l}4,22^{\mathrm{bc}} \\
\pm 0,12\end{array}$ & $\begin{array}{l}4,41^{\mathrm{bc}} \\
\pm 0,19\end{array}$ & $\begin{array}{l}4,13^{\mathrm{ac}} \\
\pm 0,12\end{array}$ \\
\hline
\end{tabular}

Signifikante Unterschiede sind durch unterschiedliche Superskripts gekennzeichnet $(\mathrm{p} \leq 0,05)$. Different superscripts show significant differences $(\mathrm{p} \leq 0,05)$.

\section{Diskussion}

Die Ergebnisse der vorliegenden Studie konnten keinen Beweis liefern, dass sich allein durch das Verlegen eines elastischen Bodens die Milchleistung einer Herde steigern 
lässt. Die durchschnittliche tägliche Milchmenge war in beiden Gruppen annähernd gleich (EBG 28,52l, BBG 28,89l, p = 0,5517). Auch der Eiweißgehalt und die Eiweißmenge zeigten keinen signifikanten Unterschied. Im Gegensatz hierzu steht der Fettgehalt der Milch, der bei der BBG signifikant über dem der EBG lag ( $<<0,0001)$. Die sich hieraus ergebende Fettmenge lag in der BBG demnach auch signifikant über der der EBG $(p=0,0002)$. Die einzige signifikante Verbesserung der Milchleistung der EBG gegenüber der BBG lag in einem signifikant niedrigeren Zellgehalt ( $\mathrm{p}<$ 0,0001). Eine Erklärung dieser Fakten könnte in der Betrachtung der Aktivitätsauswertung liegen. Laut BENZ (2002) bewegen sich Kühe auf elastischem Boden sicherer und mit mehr Vertrauen zum Untergrund. Das Lokomotionsverhalten ähnelt dem auf der Weide. Sie sind aktiver als Kühe auf Betonspaltenboden. Diese Aussage kann anhand der vorliegenden Bewegungsaktivitäts-Daten bestätigt werden. Betrachtet man in diesem Zusammenhang auch die Auswertung der Milchinhaltsstoffe getrennt nach Aktivitätsklassen, könnte sich ein logischer Zusammenhang ergeben. Mit steigender Aktivität ist ein Trend der abnehmenden Leistung zu verzeichnen. Milchmenge, Fettgehalt, Fettmenge und Eiweißmenge verzeichnen in Aktivitätsklasse 1, die den ruhigsten Tieren entspricht, unabhängig von der Aufstallung die Spitzenwerte. Zwar sind diese Unterschiede nicht signifikant, der Trend aber eindeutig. Allein der Eiweißgehalt zeigt keine Abhängigkeit von der Aktivität der Tiere. In verschiedenen Studien wurde der Beweis erbracht, dass der Blutfluss im Euter einer Kuh im Liegen im Gegensatz zum Stehen um 24-28\% erhöht ist (METCALF et al., 1992; RULQUIN und CAUDAL, 1992), was direkten Einfluss auf die Milchproduktion hat. Ein Erklärungsansatz für die signifikant niedrigere Milchleistung bezüglich des Fettgehaltes und der Fettmenge könnte demnach in der höheren Aktivität der Tiere auf dem elastischen Boden liegen. Die Annahme, dass die Tiere aufgrund der Hochboxen im Gegensatz zu den Tiefstreuboxen weniger ruhten, ist als sehr unwahrscheinlich zu bewerten. Die Optimierung der Liegeboxen durch Verlegen der komfortablen Gummimatten konnte nach Abschluss der Studie als gelungen bewertet werden, da das Problem des Spaltenliegens auf dem elastischen Spaltenboden während des gesamten Versuchszeitraumes nie auftrat und die Liegeboxen voll akzeptiert wurden. Die Beurteilung der Zellzahlergebnisse gestaltet sich komplexer. Den Haupteinflussfaktor auf den Zellgehalt stellt der auf dem Euter lastende Infektionsdruck dar (HARMON, 1994). Diesbezüglich kann in der vorliegenden Studie nicht von gleichen Voraussetzungen ausgegangen werden, da zwar die Melktechnik beider Gruppen nicht differierte, aber die Art der Liegeboxen. Hochboxen mit Gummimatten sind hier im Vergleich mit Tiefstreuboxen als hygienischer zu klassifizieren, da Verunreinigungen durch Defäkation der Tiere auf den Hochboxen größtenteils abfließen können oder manuell beseitigt wurden. Die Tiefstreuboxen hingegen wurden während des gesamten Versuchszeitraums keinerlei desinfizierender Maßnahmen unterzogen. Folgt man COOKs (2004) Definition von Kuhkomfort bei Liegeboxen, der diesen mit den Adjektiven ,sauber, trocken und komfortabel“" umschreibt, wurden diesem Anspruch die Hochboxen in der vorliegenden Studie ebenfalls eher gerecht als die Tiefboxen. Demnach bleibt eine aufstallungsbedingte, signifikante Verbesserung der Zellzahlen innerhalb der EBG festzuhalten, der Einfluss des Bodens gegenüber dem Einfluss der Liegeboxen ist jedoch nicht abzugrenzen.

Somit bestätigen die Ergebnisse der vorliegenden Untersuchung die Aussagen von BENZ (2002), dass ein elastischer Boden die Aktivität der Tiere steigert. Eine hohe 
Aktivität beinhaltet häufige Positionswechsel der Tiere, die unter optimalen Bedingungen einen Liegerhythmus von $10-12$ x täglich ca. 90 min haben (WANDEL, 2002). Diese häufigen Positionswechsel erhöhen die Bewegungsaktivität der Tiere und sind ein Indikator für einen hohen Kuhkomfortstatus (HALEY et al., 2000). Laut COOK (2004) führt aber ein höherer Kuhkomfort nicht zwingend zu mehr Leistung. Dies bestätigen auch die vorliegenden Untersuchungsergebnisse.

\section{Schlussfolgerung}

Allein durch das Verlegen von elastischem Boden im Laufstall lässt sich die Milchleistung einer Herde nicht steigern. Die Untersuchungen haben jedoch gezeigt, dass sich der Kuhkomfort dadurch merklich steigern lässt. Da Kuhkomfort die Grundlage zur Aufrechterhaltung von Gesundheit und Leistung der Herde ist (WEARY et al., 2001) und die Nachfrage des Verbrauchers nach Produkten aus „artgerechter Haltung“ stetig steigt (DE PASSILLE, RUSHEN, 1996), leistet ein elastischer Boden dennoch einen sinnvollen Beitrag zu tiergerechter Milchwirtschaft.

BAHRS, E.:

\section{Literatur}

Verhalten und Gesundheitsstatus von Mastbullen auf Gummispaltenboden. Vet. Med. Diss., LudwigMaximilians-Universität München, 2005

BENZ, B.:

Elastische Beläge für Betonspaltenböden in Liegeboxenlaufställen. Dissertation, Universität Hohenheim, 2002

BERGSTEN, C.:

Haemorrhages of the sole horn of dairy cows as a retrospective indicator of laminitis: an epedimiological study. Acta vet. scand. 35 (1994) 1, 55-66

BERGSTEN, C.:

Healthy feet requires cow comfort 24 hours. In: Proceedings of the $13^{\text {th }}$ International Symposium and $5^{\text {th }}$ Conference on lameness in ruminants, $11^{\text {th }}-13^{\text {th }}$ February, (Ed.: ZEMLJIC, B.), Maribor, Slovenija (2004)

BERGSTEN, C., FRANK, B.:

Sole haemorrhages in tied primiparous cows a san indicator of periparturient laminitis: Effects of diets,

COOK, N. B.: flooring and season. Acta vet. scand. 37 (1996), 383-94

The cow comfort link to milk quality. In Proceedings of the NMC 2004 Regional Meeting, July 29-30, Bloomongton, Minnesota, 2004

DE PASSILLE, A. M., RUSHEN, J.:

Welfare, behaviour and productivity. In: First Milky Way Meeting: Lennoxville Conference on milk production: science serving the industry - Summaries, 1996

HALEY, D. B., RUSHEN, J., DE PASSILLE, A. M.:

Behavioural indicators of cow comfort: activity and resting behaviour of dairy cows in two types of housing. Can. J. Anim. Sci 80 (2000), 257-263

HARMON, R., J.:

Physiology of Mastitis and Factors Affecting Somatic Cell Counts. J. Dairy Sci. 77 (1994), 2103-2112

HULTGREN, J., BERGSTEN, C.:

Effect of a rubber-slatted flooring system on cleanliness and foot health in tied dairy cows. Prev. vet. med. 52 (2001) 1, 75-89

KREMER, P., NÜSKE, S., SCHOLZ, A. M., FÖRSTER, M.:

Influence of different floor conditions on claw development, metabolism and milk yield in dairy cows housed in stalls with free cow traffic. In: Proceedings of the $13^{\text {th }}$ International Symposium and $5^{\text {th }}$ Conference on lameness in ruminants, $11^{\text {th }}-13^{\text {th }}$ February, (Ed.: ZEMLJIC, B.), Maribor, Slovenija, 2004

METCALF, J. A., ROBERTS, S. J., SUTTON, J. D.:

Variations in blood flow to and from the bovine mammary gland measured using transit time ultrasound and dye dilution. Res Vet Sci 53 (1992), 59-62 


\section{PARTES, N.:}

Einfluss von elastischen Laufflächen auf das Verhalten und die hygienischen Bedingungen bei Milchkühen. Diplomarbeit, Fachhochschule Weihenstephan, 2004

SAMEL, M., HEIMBERG, P., REHAGE, J.:

Growth, abrasion and hardness of claw horn and claw health of dairy cows kept on concrete or rubber mat flooring. Zweite Buiatrik-Tagung Oberschleißheim, 25.-27. Februar, Klinik für Wiederkäuer, Oberschleißheim, 2005

RULQUIN, H., CAUDAL, J. P.:

Effects of lying and standing on mammary blood flow and heart rate of dairy cows. Ann. Zootech. 41 (1992), 101

WANDEL, H., JUNGBLUTH, T., BENZ, B.:

Cow comfort in loose house systems. In: Proceedings of the $12^{\text {th }}$ International Symposium on lameness in ruminants, $9^{\text {th }}-13^{\text {th }}$ January, (Ed.: SHEARER, J. K..), Orlando, Florida, USA, 2002

WEARY, D. M., RUSHEN, J., DE PASSILLE, A. M.:

Cow comfort and barn design. In: Third Milky Way Meeting: Lennoxville conference on milk WOLF, J.: production: science serving the industry - Summaries, 2001

Haltung der Milchkuh unter Berücksichtigung hoher Milchleistungen. Arch. Tierz., Dummerstorf 43 (2000) Special Issue, 51-57

Eingegangen: 2006-01-18

Akzeptiert: 2006-02-24

Anschriften der Verfasser

PRISCA KREMER, Dr. STEFAN NÜSKE, Priv.-Doz. Dr. ARMIN M. SCHOLZ*

Lehr- und Versuchsgut der Tierärztlichen Fakultät der Ludwig-Maximilians-Universität

St. Hubertusstr. 12

85764 OBERSCHLEIßHEIM

Univ.-Prof. Dr. Dr. habil. MARTIN FÖRSTER

Institut für Tierzucht und Allgemeine Landwirtschaftslehre der Ludwig-Maximilians-

Universität

Veterinärstr. 13

80805 MÜNCHEN

*Autor für Korrespondenz, E-mail: Armin.Scholz@lvg.vetmed.uni-muenchen.de 